\title{
Self-objectification among Women Athletes and Athletic Apparel Selection
}

\author{
Anupama Pasricha ${ }^{1 *}$, Arturo Sesma, ${ }^{1}$ and Nicole Sweeney ${ }^{2}$ \\ ${ }^{1}$ Department of Apparel, Merchandising, and Design, St. Catherine University, USA \\ ${ }^{2}$ Department of Apparel, Merchandising, and Design, St. Catherine University, USA
}

Submission: February 06, 2018; Published: February 15, 2018

*Corresponding author: Anupama Pasricha, Department of Apparel, Merchandising, and Design, St. Catherine University, USA, Mail Box: 41312004 Randolph Avenue, St. Paul, MN 55105, Email: apasricha@stkate.edu

\begin{abstract}
Self-objectification can be defined as a phenomenon whereby an individual values appearance over competence. Women's self-objectification, particularly in Western societies is a normative phenomenon. This study examines self-objectification among women college athletes attending an all-women's institution. 74 women athletes at a small Midwestern university completed a four-part online Qualtrics survey. The survey included the Self-Objectification Scale, Self-Surveillance Subscale of objectified Body Consciousness (OBC) Scale, Functions of Clothing Survey, and demographic and other preferences questions. Variations in self-objectification scores were investigated across class year, sport type, and fit of upper and lower garments. Additionally, associations between self-objectification and body attitudes, clothing function, and BMI were explored. No differences across groups on self-objectification were found. Self-objectification was not found to be correlated with BMI or with various functions of clothing. However, it was positively related to the reasons for buying clothes based on fashion as well as to a preoccupation with other's perception of one's appearance. Conversely, self-objectification scores were negatively related to a woman's belief in the importance of how the body functions vs. how it appears, indicating that women in this study did not objectify their bodies. The aforementioned results are restricted to the sample of women from an all-women's institution.
\end{abstract}

Keywords: Self-objectification; Women; Athletes; Apparel

Self-objectification Among Women Athletes and Athletic Apparel Selection

Self-objectification can be defined as a phenomenon whereby an individual values appearance over competence. Self-objectification and fascination with the appearance among women is a normative and widely accepted phenomenon in the Westernized world [1]. This phenomenon has been defined through the Objectification Theory from a feminist perspective. The Objectification Theory suggests that girls and women are typically acculturated to internalize an observer's perspective as a primary view of their appearance. In patriarchal culture, this observer is typically the male [2]. Philosophers consider objectification as a morally challenging occurrence [3]. The Objectification Theory has provided illuminating connections among socio-cultural experiences, feelings about individual bodies and selves, and mental health outcomes [4]. Furthermore, self-objectification affects a wide array of appearance management behaviors [5]. Accordingly, women are trained to live up to a culturally ideal appearance and dressing is one way women manage their daily appearance. They do so by utilizing a variety of strategies including clothing selection [6].
Female college athletes have high demands for aesthetic and athletic performance, both of which contribute towards the stress and anxiety an individual experiences [7]. Several studies have examined the body image, body satisfaction, and fit of uniforms for specific sports such as basketball and golfing $[8,9]$. Empirical efforts addressing these factors may illuminate pathways toward positive body image and enhance well-being in society [6]. Also, such empirical work would highlight the role apparel plays in the everyday judgments of body image on the basis of one's clothing.

This study examines self-objectification by investigating variations in self-objectification specifically among female athletes enrolled in an all-women's 4-year university. This study is unique in its study of self-objectification and athletic apparel for all sports that are typically offered at collegiate athletic departments. It will add value to the body of knowledge in the fields of social psychology of appearance.

\section{Body image}

Body image is defined as a subjective picture of one's own appearance by self-observation and noting the reactions of 
others [10-12]. Body image is very important to the Textiles and Clothing Industry, especially in Westernized cultures because of the importance denoted to beauty and body size. It can be both perceptual and affective; it can affect the way one looks at something and how one personally feels about what he/she sees [7]. Westernized culture creates norms that are reinforced within self and have the capacity to distort body image. This negative view of one's body may cause one or more disorders that affect the health of individuals [7]. While this is a potential threat to any individual, it is especially dangerous to athletes at the elite or collegiate levels. Because of the performance demands that come from the audience, the athlete, and the sport itself, athletes may experience body dissatisfaction. There is the idea that weight along with fat reduction, will enhance athletic performance. Coaches and athletes alike encourage this ideal. Athletes are at a greater risk of eating disorders than nonathletes $[7,13]$. Scholars also note that it is more common for females to experience body dissatisfaction and associated risks than men [7], it can be anywhere from $75 \%-95 \%$ for women and $33 \%-45 \%$ for men [14].

\section{Objectification theory}

Fredrickson \& Roberts [15] postulated The Objectification Theory. This theory asserts that the cultural practices of heterosexuality, media images with men gazing at women, and specific vocabulary encourages sexual objectification of women. This sexual objectification builds an internalized view of self as an object and a constant self-monitoring pattern, which may lead to negative psychological consequences and mental health risks [15]. The Objectification Theory is formulated from the social construction of women's bodies through sociocultural practices that focus on sexual objectification and measured display of femininity through movement and dress [1].

All women are not equally affected by self-objectification; it varies with age, ethnicity, sexuality, and personal context. There is evidence that self-objectification is a common practice among women in westernized societies. In such societies, the media places immense pressure on women for meeting societal appearance ideals $[6,16]$. This is conflicting because self-objectification is defined as the process of internalizing the outsider's or observer's view [5]. Objectification presents one's body as a separate entity from oneself. This perspective allows the body to be compared to a common object: identifiable but lacking personable characteristics. Socially constructed gender power differential empowers men to gaze at women and oppresses women by comparing them to an object for use and desire. This may have a considerable effect on women [17].

Women constantly try to achieve socially desirable objectified bodies. There are several studies that state women exercise to change their body appearance. Even if women are unable to notice any tangible physical change after exercise, they often feel better after working out. The process of building strength and endurance is not only achieved by the body, but the individual as well. There is pressure from the media and culture to engage in physical activity in order to lose weight and boost sexual appeal. Kennedy \& Markula [18] argue that this is a feminizing activity because it sets women apart from men. Working to lose excess weight and increase sex appeal can be a never-ending journey. Therefore, the ideal fit and feminine body may actually be unachievable for some individuals.

The media encourages self-objectification behaviors and disguises them as acceptable and unquestionable to ensure that the feeling of being objectified is not foreign to women of this culture and era. Feeling ashamed of the appearance and/or ability of one's body can emerge from evaluating it based on a cultural ideal standard. This standard manifests itself in the form of the images that surround us every day. The Objectification Theory lens connects socio-cultural experiences, feelings about one's bodies and the resulting mental health outcomes [4]. These objectified views of an individual's body may influence her body satisfaction and increase body consciousness in a negative way. They could also lead to over-awareness of one's body image and its ability.

When supporting the movement towards facilitating a positive body image, it is important to consider the manner in which people manage objectifying. Past research illustrates that athletes are a particularly vulnerable population for body image related issues [6]. Clothing can often be used as a way to how people view athletes and how athletes present themselves to others, how they desire to be viewed, and how society expects athletes to look. Gurung \& Chrouser [17] discuss that "female athletes are largely perceived as able-bodied, talented women and are strong prototypes of determination and character" (p. 93). This statement elucidates a brief overview of the common pressures that women collegiate athletes face. The burden to meet these expectations, coupled with being objectified can be stressful. An individual's confidence of her body often dictates the strength and power she is expected to have as an athlete. This self-ideal view is compromised if the athlete feels she is unable to meet these expectations.

Studying athletes' perception of their sports apparel or uniform clad body and what they value most about their apparel can illuminate their preference of garments. The fit of the garments is vital in providing an encouraging and healthy atmosphere for these athletes to compete in. Athletic apparel can have a variety of fits and styles that are based on the particular kind of sport. Tight and revealing clothes may heighten the level of self-surveillance and objectification. For example, Tiggeman \& Lacey [19] reported that the individual who wore tighter exercise clothes and fashionable gym clothes had a higher level of self-objectification. Furthermore, athletic wear has followed the fashion trends of the particular time period [20].

\section{Functions of apparel}

Many theoretical frameworks have been applied to understand apparel selection based on the functions it serves. Apparel provides for the physical, psychological, and sociocultural 
needs of human beings [21]. Moreover, apparel choices are also based on perceived body image by individuals [12]. In a study by Feather, et al. [22], perceived satisfaction with the body was associated with perceived satisfaction with clothing. A study was conducted to find the relationship between self-objectification and appearance management behavior where the behavior can range from routine behavior of apparel selection to extreme behavior of surgical body modification [5]. Tiggemann \& Andrew [23] performed a study with undergraduate students to assess clothing selection and body image. They found that BMI and self-classified weight were positively correlated with the choice of clothes for camouflage. Furthermore, they reported that selfobjectification was positively correlated with choice of clothes for fashion, and negatively correlated with choice of clothes for comfort. Dickson \& Pollack reported similar results from their study on in-line skaters where skaters preferred fashion over comfort (2000). In another study, Prichard \& Tiggemann [24] found that garment fit and style preference were correlated to self-objectification and surveillance. A study of female collegiate basketball players revealed that satisfaction with the upper body was higher than with the lower body [8]. Labat \& Delong [25] asserted that measure of fit satisfaction was positively correlated with Body Cathexis (Body Cathexis is the extent of satisfaction with parts and abilities of the body). Their findings were consistent with both upper and lower body, and the total body in female consumers. Body Cathexis has been found to be lowest for the midsection of the body [12].

\section{Athletes}

Athletes undergo stress and anxiety of appearance and fitness level as an everyday life norm. High demands of performance can fuel an athlete in both positive and negative ways and can have significant influence on the outcome of their performance. The nature of their competitive activity often exerts pressure on the athlete. They must perform at their optimal at all times, often in front of a large audience. They must never cease to improve, both in competition and in training. While developing their athletic ability, they simultaneously ought to maintain the ideal physique society expects from them. This can be an exhausting combination of expectations to maintain, performing close to perfection whilst looking the part of being in impeccable athletic condition. The media portrayal of what an athlete "should" look like may lead to dissatisfaction with an athlete's own body image, even if he or she is in a physically peak athletic condition. On the contrary, with positive feelings about body image, a female athlete has the capacity to tap into an entirely new arena of enhanced athletic achievement. An important tool in facilitating positive body image is the apparel and uniform that these athletes wear.

Based on Fredrickson's \& Roberts [15] Objectification Theory and past research, this study examines the links between selfobjectification and athletic apparel selection among collegiate women athletes at an all-women's college. It is guided by the following objectives: a. Determine overall trait self-objectification,

b. Examine differences in self-objectification between under and upper-class women,

c. Examine differences in self-objectification in individual and team play, and

d. Explore the link between athletic apparel selection and self-objectification of collegiate women athletes.

\section{Method}

\section{Participants}

Survey data from 74 athletes from a private all-women Midwestern university participating in varsity teams of various sports were collected. The sports included basketball, crosscountry, dance, golf, hockey, soccer, softball, swimming \& diving, tennis, track \& field, and volleyball. Participants were recruited through the respective athletic team coaches via the Department Chair of Nutrition and Exercise Science Department at the University. This recruitment took place via an email announcement followed by individual consultation between the coaches and the Department Chair. Participants were primarily Caucasian (93\%) and between the ages of 18-22. All years of the undergraduate study were represented in the study: firstyear(27\%), sophomore (30\%), junior (13\%), senior $(27 \%)$, and other $(3 \%)$.

\section{Materials}

The survey completed by participants was segmented into 4 parts: Self-Objectification Scale (SOQ), Objectified Body Conscious Scale (OBC), Functions of Clothing Survey, and demographic and other preferences questions. The main outcome variable for this study comes from the SOQ [26]. This scale assesses the degree to which people view their bodies in terms of performance and health versus appearance. Participants were asked to rank order 10 body attributes, with zero being considered the least important to 10 as the most important. These attributes were divided into two categories: competence and appearance. The rankings of the five competence attributes (health, strength, physical coordination, physical fitness, and energy level) and the rankings of the five appearance attributes (firm/sculpted muscle, sexual appeal, weight, physical attractiveness, and body measurements) were added up; these two scores were then subtracted from each other to calculate the self-objectification score. Self-objectification scores range from -25 , which project to a healthy perspective of low selfobjectification and an emphasis on body competence, to a +25 , which reflects a high value on self-objectification and attitudes that are focused on body appearance. Validity of this scale has been tested and reported by Noll \& Fredrickson [26].

In the second part, another index of how one perceives her body was assessed using a modified approach to McKinley \& Hyde's [27] 8-item Objectified Body Consciousness (OBC) scale. This scale aims to measure the extent to which women have 
internalized the cultural standard of women's body. Although McKinley \& Hyde [27] report three reliable and valid scales of surveillance (viewing the body as from an outside perspective), body shame (when the body does not meet certain expectations) and appearance control beliefs. This study used the Surveillance Subscale and was unable to replicate its reliability. Therefore, a principal-component analysis was used to identify empirically guided scales within the surveillance subscale, and two robust and interpretable scales were found. The first is referred to as body-looks, and is comprised of four items that assess the participants relative disregard for how other people view them (e.g., "I rarely think about how I look" or "I often worry about whether the clothes I am wearing make me look good" [reverse coded]). The second scale is named body-feels, and is comprised of two items that focus on the functions and performance of the body rather than how it looks ("I think more about how my body feels than how my body looks" and "I am more concerned with what my body can do than how it looks"). Each scale showed acceptable internal consistency using Cronbach's alpha, with the body-feels $\alpha=.73$ and the body-feels $\alpha=.61$.

In the third part of the survey, justifications behind individuals purchase of clothes was assessed using the 15-item Functions of Clothing Survey [24], which yielded four scales. The assurance scale assessed the degree to which a person buys clothes to boost self-confidence (e.g., "I select clothes that make me feel better;" 5 items, $\alpha=.84$ ); the individuality scale measured the extent to which a person buys clothes that are distinctive, or make one stand out (e.g., "I select clothes that are exciting;" 4 items, $\alpha=.71$ ); the camouflage scale measured whether a person buys clothes to hide their body (e.g., "I select clothes that do not call attention to my figure;" 3 items, $\alpha=.66$ ); and the fashion scale assessed the degree to which someone will purchase clothes that are stylish and for appearance's sake (e.g., "I select clothes that will impress people;" 3 items, $\alpha .84$ ).

Finally, demographic and other clothing related preference questions were asked in the last section of the survey. Respondents indicated their age, class status (freshman, sophomore, junior, or senior), race/ethnicity, height, weight, the college-level sports they play, and their preference for garment fit. Height and weight were used to calculate body mass index (BMI). Sports participation was coded as either participant of an individual or a team sport; Golf, swimming, and tennis were coded as individual, while soccer, softball, and volleyball were coded as team sports. These were mutually exclusive categories, any respondents who participated in both types of sports or in neither type $(\mathrm{N}=14)$ were excluded. Finally, participants were asked to indicate which type of athletic garment fit they preferred: loose, semi, or tight. This question was asked regarding both upper garments and lower garments.

\section{Procedure}

The survey was administered using Qualtrics online survey tool available through the IT services of the university. The online survey link was emailed to participants through the respective athletic team coaches. An IRB approved email with a link to the survey was emailed to the Department Chair. The chair used the email text to consult with and request coaches support in encouraging their athletes to participate. In the email text and the online survey, participants were informed that the survey was anonymous and participation voluntary. They were also informed that by submitting their answers to the online survey they are giving consent for data to be used for research. Password protected data was stored on Qualtrics system. Only the researchers have access to the data. The data will be deleted after two years from the completion of the study. Descriptive as well as inferential statistical analysis of 74 surveys is discussed in the following section.

\section{Results}

\section{Descriptive statistics}

Overall, women in this study reported relatively healthy attitudes regarding the self-objectification of their bodies: $\mathrm{M}=$ $-3.7(\mathrm{SD}=14.0)$. This negative mean value indicates that women prioritized attributes such as health, strength, and energy level, considered to be competent attributes by Fredrickson et al. over appearance attributes, such as sexual appeal, physical attractiveness, and body measurements. Figure I displays the distribution of scores for the sample and demonstrates that while the full -25 to +25 range was covered, the preponderance of scores are in the negative (i.e., healthier) territory.

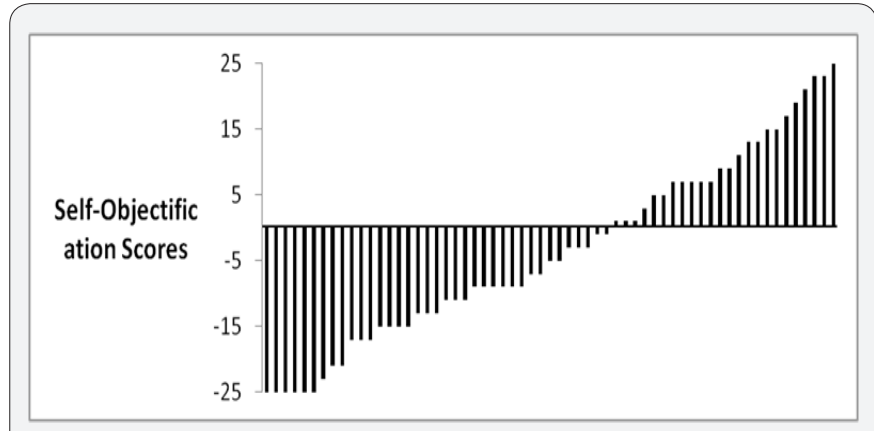

Figure 1: Distribution of Self-Objectification Scores.

Women's BMI values were calculated to be 23.0 ( $\mathrm{SD}=3.4$ ), within the Centers for Disease Control's range of a normal BMI18.5-24.9 (Centers for Disease Control and Prevention, n.d). With respect to the functions of clothing, the mean values for the 4 scales were all between 2.2 and 2.8 (Assurance- $M=2.2$, $S D=.55$; Fashion $-M=2.3, S D=.81$; Individuality $-M=2.6, S D=.60$; Camouflage $-\mathrm{M}=2.8, \mathrm{SD}=.80$ ), indicating that for each function, the sample tended to select clothes for themselves that satisfied each of these clothing purposes. Finally, participants' mean values for the surveillance scales of how the body feels and looks were calculated. The mean for how the body-feels scale was $2.8(\mathrm{SD}=.79)$, suggesting that the sample was more likely to endorse attitudes regarding the importance of how their body feels over how their body looks. Similarly, the mean value for the how the body-looks scale was $3.4(\mathrm{SD}=.65)$, indicating that 
women were more likely to disagree with statements endorsing the importance of how other people view, judge, and appraise one's appearance.

\section{Group differences}

T-tests and ANOVAs were conducted in order to examine any differences across groups on self-objectification scores. In particular, the study examined whether the self-objectification scores would differ by class status (freshman/sophomores vs. juniors/seniors), type of sport (individual sport vs. team sport), and how tight or loose women preferred their athletic clothing to fit. Table I lists the means, standard deviations, and tests of differences to analyze each of these comparisons.

Table 1: Means, SDs and Significance Levels Across Groups on Self-Objectification.

\begin{tabular}{|c|c|c|c|c|}
\hline & \multicolumn{2}{|c|}{ Self-Objectification Score } & \multirow[b]{2}{*}{ Test Statistic } & \multirow[b]{2}{*}{ Sig. } \\
\hline & $M$ & $S D$ & & \\
\hline \multicolumn{5}{|l|}{ Class Status } \\
\hline $1^{\text {st }} /$ Soph. Year & -4.25 & 14.3 & \multirow{2}{*}{$t(72)=0.62$} & \multirow{2}{*}{ ns } \\
\hline Jr/Sr Year & -1.87 & 14.4 & & \\
\hline \multicolumn{5}{|l|}{ Sport Type } \\
\hline Individual & -3.97 & 13.3 & \multirow{2}{*}{$t(72)=2.45$} & \multirow{2}{*}{ ns } \\
\hline Team & -2.77 & 14.2 & & \\
\hline \multicolumn{5}{|c|}{ Garment Fit - Upper Body } \\
\hline Loose & -.71 & 17.3 & \multirow{3}{*}{$F(2.56)=0.55$} & \multirow{3}{*}{ ns } \\
\hline Semi & -5.48 & 13.3 & & \\
\hline Tight & -2.88 & 13.2 & & \\
\hline \multicolumn{5}{|c|}{ Garment Fit - Lower Body } \\
\hline Loose & -1.0 & 14.9 & \multirow{3}{*}{$F(2.56)=1.52$} & \multirow{3}{*}{ ns } \\
\hline Semi & -8.10 & 13.9 & & \\
\hline Tight & -1.58 & 13.8 & & \\
\hline
\end{tabular}

Note: Self-objectification scores range from -25 to +25 , with negative values indicating healthier attitudes towards one's body.

For class status, first-year and sophomore students reported statistically similar values of self-objectification $(\mathrm{M}=-$ $4.25, \mathrm{SD}=14.3)$ as did juniors and seniors $(\mathrm{M}=-1.87, \mathrm{SD}=14.4)$; $\mathrm{t}(56)=0,62, \mathrm{p}=.54,95 \%$ CI $[-10.09,5.32]$. For sport type, participants in individual sports $(M=-3.97, S D=13.3)$ and team sports $(M=-2.77, S D=14.3 ; t(56)=0.32, \mathrm{p}=.78,95 \%$ CI $[-6.22$, 8.61]) reported similar self-objectification scores. For both upper and lower body garment fit, women who prefer semi-tight fighting garments had lower self-objectification scores. Women

\section{Correlations}

Table 2: Correlations among Self-Objectification Scores and BMI, Clothing Functions, and Surveillance.

\begin{tabular}{|c|c|}
\hline & Self-Objectification Score \\
\hline BMI & -.06 \\
\hline Functions of Clothing & \\
\hline Assurance & -.13 \\
\hline Individuality & .00 \\
\hline Camouflage & -.16 \\
\hline Fashion & $.31^{*}$ \\
\hline Body Surveillance & \\
\hline Body Looks & $.40^{* * *}$ \\
\hline Body Feels & -.51 \\
\hline
\end{tabular}

Note. Lower self-objectification scores are considered healthier attitudes to possess. BMI= body mass index.

${ }^{*} p<0.05 ;{ }^{* *} p<0.001$. who prefer loose-fitting garments had higher There were no differences in self-objectification scores across garment fit for either upper body or lower body clothing: upper body garment fit: $F(2,56)=0.55, \mathrm{p}=.58, \eta_{p}^{2}=.02$; lower body garment fit: $\mathrm{F}(2,56)=1.52, \mathrm{p}=.23, \eta_{p}^{2}=.05$ (Table 1$)$.

Note. Self-objectification scores range from -25 to +25 , with negative values indicating healthier attitudes towards one's body.

Finally, zero-order correlations were explored between selfobjectification scores and attitudes towards one's clothing, one's body surveillance, and one's BMI; these correlations are presented in Table 2. Self-objectification scores were not statistically related to BMI ( $\mathrm{r}=.06)$, Assurance ( $\mathrm{r}=.13)$, Individuality ( $\mathrm{r}=.00)$, or Camouflage ( $\mathrm{r}=-$-16).. Self-objectification scores were moderately positively correlated with the clothing function Fashion $(\mathrm{r}=.31, \mathrm{p}<.05)$, indicating that the more likely women were to endorse that they purchase clothes that are fashionable, stylish, and to impress people, the more likely they were to value body attributes such as sexual appeal, body measurements, and physical attractiveness. Likewise, self-objectification scores were moderately positively correlated with the surveillance scale of body looks $(\mathrm{r}=.41, \mathrm{p}<.001)$, suggesting that women who report that they are preoccupied with how they are viewed by other people are also more likely to endorse higher values of self-objectification. Finally, self-objectification scores were moderately negatively related to the surveillance scale body 
feel $(r=.51, p<.001)$, indicating that women who are concerned more about how their body feels and performs than with how it looks are also more likely to believe in the importance of body attributes such as health, strength, and energy levels.

\section{Discussion}

The purpose of the study was to investigate self-objectification and athletic apparel selection among collegiate women athletes at an all-women's college with following specific objectives: a) to determine overall trait self-objectification, b) to examine differences in self-objectification between under and upperclass women, c) to examine differences in self-objectification in individual and team play, and d) to explore the link between athletic apparel selection and self-objectification of collegiate women athletes.

Overall, women in this study reported relatively healthy attitudes regarding the self-objectification of their bodies. First, the mean value for self-objectification was negative, indicating that overall, this sample tended to prioritize body competence over body appearance. Likewise, the women in this study reported this same emphasis on what their body can do through their relative disregard for how they appear to other people in the surveillance scales of body-feels and body-looks. Second, there were no differences in self-objectification scores across any of the examined groups; different class levels, sports types, and garment preferences demonstrated similar low levels of importance on sexual appeal, physical attractiveness, and body measurements. Additionally, self-objectification was unrelated to BMI. Finally, the study did find that self-objectification was correlated in coherent ways with body attitudes and clothing function: women who tended prioritized fashion, appearance, and looks tended to report higher levels of self-objectification. These correlations provide further evidence of the utility of the validity of self-objectification and self-surveillance in a variety of samples [28,22]. These findings resonate with past research documenting the association between clothing preference and body image, body size [29] and also self-objectification [23], as well as the lack of correlation between self-objectification and BMI [14].

On the contrary, some of the results of this study disagree with past research. For example, Tiggeman \& Lacey [19] reported an association between self-objectification and the tightness of fit in exercise and gym clothes, while we found no relation between self-objectification and upper or lower body athletic apparel selection. It seems likely that there is a meaningful difference between clothes that women wear "out to the gym" vs. apparel women athletes wear while participating in an organized sport. This distinction bears further exploration.

Perhaps the largest departure between this study's findings and past research is in the relatively healthy attitudes women possessed towards their bodies. Past research has reported high levels of self-objectification in samples of women [30,31]. This discrepancy in findings between the low self-objectification scores and positive body image in this sample vs. past research may be attributed to the social context in which this study was conducted, sample size, and voluntary nature of participation. Every woman in this study attends an all-women's baccalaureate college whose institutional mission focuses on teaching women to "lead and influence" (XXX University Mission \& Vision, n.d.). Anecdotal evidence from discussions with students suggests that the students attending this all-women's institution feel more comfortable and less pressured to follow cultural norms of ideal body because there are no men. While the study did not probe causal mechanisms, it seems likely that the institution's efforts to reduce pressure on women to self-objectify, and encourage accomplishment and self-empowerment, combined with the absence of men in classrooms and on campus have had an effect on the degree to which these women self-objectify. Furthermore, our sample is comprised of all athletes; this could attribute to the diminished self-objectification, thereby augmenting the importance of body function, as these are women wellconditioned for their respective sports. Competence in this activity may provide sufficient validation for one's body image that may otherwise be required in other domains (e.g., body appearance and fashionable clothes) [32].

\section{Implications of findings for the objectification theory}

Our findings indicate that we cannot generalize the Objectification Theory for all women. Our sample had positive body image and low self-objectification scores. Therefore, we recommend that in a study of the Objectification Theory and selfobjectification, researchers should carefully analyze contextual factors and incorporate them in their results and discussion. With specific reference to this study, these factors include the absence of male impressions as it is an all-women's school, and the institutional philosophy to empower women. These implications parallel those reported in Fredrick, et al. [22], only the factors were different.

\section{Limitations and future direction}

As the participants of this study are from an all-women's baccalaureate college and the sample size is small, the results of the study are not generalizable. Another major limitation in this study is the possibility of bias. The utilized method was a voluntary self-selected manner of participation and the complete population of athletes may not have been represented (however, sample represented $40 \%$ of the population), thereby, potentially skewing the data. We recommend a participation method for full representation. Furthermore, we also recommend examining and clarifying the potential effects of a co-ed vs. all women college experiences on self-objectification for both athletes and non-athletes. The Objectification Theory suggests that females are typically acculturated to internalize an observer's (male) perspective as a primary view of their appearance. This may influence the objectification scores of female athletes from a co-ed institution. Therefore, we recommend that future 
researchers replicate this study at a co-ed institution and use methodology that can garner higher response rate. This will further clarify the role of social context and athletic involvement on self-objectification. Furthermore, researchers can expand the study on a larger sample to generalize findings that explore the relationship between the self-objectification, role of apparel, and athletic performance.

\section{References}

1. Calogero RM, Tantleff-Dunn S, Thompson JK (2011) Objectification theory: An introduction. In: Calogero RM, Tantleff-Dunn S et al. (Eds.) Self-objectification in women. Washington D.C: American Psychological Association, USA pp. 3-21.

2. Bartky S (1990) Femininity and domination: Studies in the phenomenology of oppression. New York, NY: Routledge, USA.

3. Papadaki E (2012) Feminist perspectives on objectification. In: Zelta EN (Eds.), The Stanford encyclopedia of philosophy (winter 2012 edition).

4. Moradi B, Huang Y (2008) Objectification theory and psychology of women: A decade of advances and future directions. Psychology of Women Quarterly 32(4): 377-398.

5. Lee J, Johnson KKP (2009) Factors related to engagement in risky appearance management behaviors. Clothing and Textiles Research Journal 27(3): 163-178.

6. Dickson MA, Rudd NA, Lennon SJ (2006) Focused social responsibility: Part 2. Clothing and Textile Research Journal 24(4): 279-281.

7. Rudd NA, Carter J (2006) Building positive body image among college athletes: a socially responsible approach. Clothing and Textiles Research Journal 24(4): 363-338.

8. Feather BL, Ford S, Herr DG (1996) Female collegiate basketball players' perceptions about their bodies, garment fit and uniform design preferences. Clothing and Textiles Research Journal 14(1): 22-29.

9. Wheat KL, Dickson MA (1999) Uniforms for collegiate female golfers: Cause for dissatisfaction and role conflict? Clothing and Textiles Research Journal 17(1): 1-10.

10. Body image (2014) In Merriam-Webster.com.

11. Fallon A (1990) Culture in the mirror: Socio cultural determinants of body image. In: Cash TF \& Pruzinsky T (Eds.), Body images: Development, deviance, and change. Guilford, New York, USA, pp. 80109.

12. Kaiser SB (1997) The social psychology of clothing. Fairchild Publications, New York, USA.

13. Smolak L, Murnen SK, Ruble AE (2000) Female athletes and eating problems: a meta analysis. Int J Eat Disord 27(4): 371-380.

14. Grogan S (1999) Body image: Understanding body dissatisfaction in men, women, and children. Routledge, London, UK.

15. Fredrickson BL, Roberts TA (1997) Objectification theory: Toward under-standing women's lived experiences and mental health risks. Psychology of Women Quarterly 21(2): 173-206.

16. Poorani A (2012) Who determines the ideal body? A summary of research findings on body image. New Media and Mass Communication p. 2.

17. Gurung RAR, Chrouser CJ (2007) Predicting objectification: do provocative clothing and observer characteristics matter? Sex Roles 57(1-2): 91-99.

18. Kennedy E, Markula P (2011) Women and exercise: The body, health and consumerism. Routledge, New York, USA.

19. Tiggemann M, Lacey C (2009) Shopping for clothes: Body satisfaction, appearance investment, and functions of clothing among female shoppers. Body Image 6(4): 285-291.

20. Klepp I, Sørheim S, Enstad K (2012) Sports and Dress. In Berg Encyclopedia of World Dress and Fashion: Volume 8-West Europe.

21. Marshall SG, Jackson HO, Stanley M Sue (2012) Individuality in clothing selection and personal appearance. Upper Saddler River, NJ: Prentice Hall, US.

22. Fredrick DA, Forbes GB, Grigorian KE, Jarcho JM (2007) The UCLA body project I: Gender and ethnic differences in self-objectification and body satisfaction among 2,206 undergraduates. Sex Roles 57(5-6): 317-327.

23. Tiggemann M, Andrew R (2012) Clothing choices, weight, and trait self-objectification. Body Image 9(3): 409-412.

24. Prichard I, Tiggemann M (2005) Objectification in fitness centers: Selfobjectification, body dissatisfaction, and disordered eating on aerobic instructors and aerobic participants. Sex Roles 53(1-2): 19-28.

25. Labat KL, DeLong MR (1990) Body cathexis and satisfaction with fit of apparel. Clothing and Textiles Research Journal 8(2): 43-48.

26. Fredrickson BL, Roberts TA, Noll SM, Quinn, DM, Twenge JM (1998) That swimsuit becomes you: Sex differences in self-objectification, restrained eating, and math performance. J Pers Soc Psychol 75(1): 269-284.

27. McKinley NM, Hyde JS (1996) The Objectified Body Consciousness Scale: Development and validation. Psychology of Women Quarterly 20(2): 181-215.

28. Calogero RM (2011) Operationalizing self-objectification: Assessment and related methodological issues. In: Calogero RM, Tantleff-Dunn S \& Thomson JK (eds.), Self-objectification in women American Psychological Association, Washington DC, USA pp. 23-49.

29. Chattaraman V, Rudd NA (2006) Preferences for aesthetic attributes in clothing as a function of body image, body cathexis and body size. Clothing and Textiles Research Journal 24(1): 46-61.

30. Centers for Disease Control and Prevention (n.d). About BMI for adults.

31. Dickson MA, Pollack A (2000) Clothing and identity among female inline skaters. Clothing and Textile Research Journal 18(2): 65-72.

32. Mission and Vision (n.d). Retrieved from http://www.stkate.edu/ pages/aboutstkates/women.php. 
Your next submission with Juniper Publishers will reach you the below assets

- Quality Editorial service

- Swift Peer Review

- Reprints availability

- E-prints Service

- Manuscript Podcast for convenient understanding

- Global attainment for your research

- Manuscript accessibility in different formats ( Pdf, E-pub, Full Text, Audio)

- Unceasing customer service

Track the below URL for one-step submission https://juniperpublishers.com/online-submission.php 